\title{
An MHT algorithm for UWB radar-based multiple human target tracking
}

\author{
SangHyun Chang ${ }^{1,2}$, Michael Wolf ${ }^{1}$, and Joel W. Burdick ${ }^{1}$ \\ ${ }^{1}$ Engineering \& Applied Science, California Institute of Technology, MC 104-44, Pasadena, CA \\ ${ }^{2}$ Department of Electrical Engineering - Electrophysics, University of Southern California, Los Angeles, CA \\ \{sanghyun,wolf\}@ caltech.edu, jwb@ robotics.caltech.edu
}

\begin{abstract}
This paper presents a multiple hypothesis tracking (MHT) framework for tracking the ranges and velocities of a variable number of moving human targets via a monostatic ultra-wideband (UWB) radar. The multi-target tracking (MTT) problem for UWB radar-based human target tracking differs from traditional applications because of the multitude of observations (multipath scattering) per target in each scan, due to the short spatial extent of the transmitted UWB signal pulse width. We develop an MHT framework for UWB radar-based multiple human target tracking that extends a previously studied human tracking algorithm. We present experimental results in which a monostatic UWB radar tracks both individual and multiple human targets, even with changing numbers of targets across radar scans.
\end{abstract}

Index Terms-UWB radar, tracking, filtering, multi-target tracking, multiple hypothesis tracking

\section{INTRODUCTION}

This paper introduces a multiple hypothesis tracking (MHT) framework for tracking the ranges and velocities of a variable number of moving human targets via a mono-static ultrawideband (UWB) radar. Because the ability to track human movement is useful for the wide range of security and safety applications, a number of technologies have been developed for human tracking. Computer vision has limited performance in poor visibility conditions, while the performance of infrared imagers can be temperature dependent. Human LADAR signatures are often not highly discriminable from other moving clutter, and LADAR performance degrades in dusty and foggy conditions. UWB radar can provide a complementary technology for detecting and tracking humans, particularly in poor visibility or through-wall conditions. Building upon our previous work [1], this paper presents an algorithm for solving data segmentation and data association problems in tracking a variable number of human targets with UWB radar.

Compared with RF, microwave, and mm-wave radar [2], [3], UWB radar provides high-resolution ranging and localization due to the fine temporal resolution afforded by wide signal bandwidth [4], [5], [6]. In our previous work, Chang, et. al., developed an Expectation-Maximization Kalman Filter (EMKF) algorithm for UWB radar-based tracking a fixed number of humans. However, since this algorithm assumes a fixed number of targets to track, it is necessary to develop a multitarget tracking (MTT) solution which allows for changing

The authors greatly appreciate the financial support of this work provided by the LIG Nex1 Corporation, Yongin, Korea. numbers of targets across radar scans, false measurements (clutter), and missed detections (temporary occlusions).

An abundant MTT literature has considered military radar and computer vision tracking applications [7], [8], [9]. However, the key differentiator of MTT for UWB radar-based tracking versus traditional applications is the multitude of observations (multipath scattering) per target in each scan, due to the short spatial extent of the transmitted UWB signal pulse width [10]. Wolf recently developed a multi-hypothesis cluster tracking (MHCT) algorithm for sorting and tracking extracellular neural recordings [11]. Based on the cluster-like nature of both neural spike observations and UWB multipath scatter, we develop a variant of Wolf's algorithm for UWB radar-based multiple human target tracking problem, which extends our previously developed algorithm to the more realistic case of varying target number.

Section II summaries the simple multipath signal model that underlies our approach, and shows that scattering paths' time-of-arrival (TOA) can be interpreted as a point process governed by a Gamma distribution. Section III reviews our previous EMKF tracking algorithm for a fixed number of targets. Section IV presents our proposed MHT algorithm for tracking a variable number of humans, while Section V presents experimental results to illustrate our approach.

\section{UWB Signal Modeling}

\section{A. UWB Scattered Waveform Model}

The UWB radar signal scattered from a human body includes multiple path components, as the impinging UWB electromagnetic wave scatters from different human body parts at different times with various amplitudes (depending on the distance to the body part, and the size and material of the reflecting part). Thus, the returned UWB radar signal $w(t)$ can be approximated by a specular multipath model [12], [13]:

$$
w(t) \approx \sum_{j} a_{j} p\left(t-n_{j}\right),
$$

with $a_{j}$ and $n_{j}$ respectively representing the amplitude and time-of-arrival (TOA) of the $j^{\text {th }}$ component of the received signal, and $p(t)$ is an elementary waveform shape, e.g., the transmitted radar waveform in free space. For example, the waveform is recorded over an interval $t \in\left[t_{0}, t_{\max }\right]$, which corresponds to a range of $r \in\left[r_{0}, r_{\max }\right]=\left[c t_{0} / 2, c t_{\max } / 2\right]$, where $c$ is the speed of light (see details in [13]). The specular 
multipath model is an approximation whose simplicity allows for real-time processing without compromising UWB radar's high time-resolution capability. Each path's TOA and amplitude can be estimated by the CLEAN algorithm with a given waveform template [13], [14].

Since UWB radar scatters from both stationary and moving objects, all scatters obtained from a complex test environment must be analyzed for human target candidates. To reduce the high computational cost associated to such analysis, a moving target indication (MTI) system, summarized in [13], is used to eliminate highly human-unlike scatters.

\section{B. Human Scattered Waveform Characterization}

In order to understand the basic scattering behavior, we constructed a database of UWB radar scans obtained while a human walked randomly in an open field within the vicinity of the radar (see details in [1]). The radar returns were calibrated and processed using the CLEAN algorithm to extract the amplitudes and TOAs of the scattering components. These returns were then manually segmented to ensure a correct data association between detected scatter paths and the human target. To characterize scattered waveforms from moving humans, we introduce two variables: human range and adjusted time-of-arrival. The human target's nominal range is defined as the first moment of the power range profile $r$ [13]:

$$
r=\frac{\sum_{j \in \Omega} R_{j}\left(a_{j} R_{j}^{2}\right)^{2}}{\sum_{j \in \Omega}\left(a_{j} R_{j}^{2}\right)^{2}},
$$

where $R_{j}=\left[n_{j} \cdot c\right] / 2$ is the $j^{\text {th }}$ scattering path's range ${ }^{1}, n_{j}$ is the TOA of the $j^{t h}$ scatter component, and $\Omega$ is a set of path indices associated with the human target. It is convenient to introduce an adjusted TOA (ATOA) variable:

$$
\delta_{j}=R_{j}-r+K,
$$

where $r$ is the range to the human, and $K$ is a constant offset related to the delay spread of a typical human. Then, the mono-static UWB radar scattering process for walking humans, under the specular multipath model in Equation (1), can be interpreted as point process governing the ATOAs. After studying common univariate distributions, we found that the ATOA histogram was best fit by a Gamma distribution whose mode lies at the human target location and whose probability density function $(\mathrm{PDF}) f_{\Gamma}(\delta ; \kappa, \theta)$ is:

$$
f_{\Gamma}(\delta ; \kappa, \theta)=\delta^{\kappa-1} \frac{\exp (-\delta / \theta)}{\theta^{\kappa} \Gamma(\kappa)} \text { for } \delta>0,
$$

where $\Gamma(\cdot)$ is the Gamma function, and $\kappa, \theta$ are respectively the Gamma distribution's shape and scale parameters ${ }^{2}$. In our application, the $\kappa$ parameter is a fixed value characteristic of humans, which is estimated from the database at $\kappa=7.60$ in Figure 1. The $\theta$ parameter is related to target location, and is estimated during the tracking process. While our choice of the Gamma distribution was based on an empirical study, we note

\footnotetext{
${ }^{1}$ We refer to TOA and range interchangeably in the paper

${ }^{2}$ All empirical ATOA are adjusted to be positive with $K=0.533 \mathrm{~m}$.
}

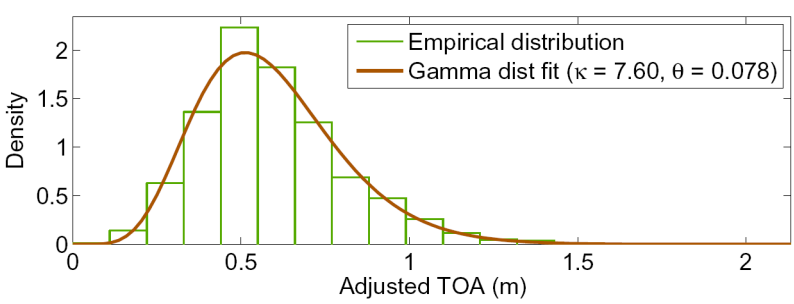

Fig. 1. Histogram of multi-path ATOA, with Gamma distribution fit.

that the Gamma distribution exactly models the distribution of arrival times for Poisson distributed events. It is thus a plausible model for human scatter ATOAs.

\section{TRacking a FiXed Number of Human TARgets}

For simplicity of exposition, this section summarizes our prior work [13], which forms the basis for the new developments of this paper. First we show how to track a fixed number of humans using an Expectation-Maximization Kalman Filter (EMKF) algorithm, where the EM algorithm simultaneously associates individual scatter paths to each target and estimates each target's state. The next section, which represents the new contributions of this paper, shows how to add a multiple-target tracking (MTT) capability to this framework via the use of a cluster-based multiple-hypothesis tracking (MHT) procedure.

We define the state vector $\mathbf{x}$ of a human target as $\mathbf{x}=[r v]^{T}$, where $r$ and $v$ respectively denote the range and velocity (time rate of change of the range) of the human target, and $(\cdot)^{T}$ denotes the transpose. For simplicity, we use a simple random walk model to model human dynamics:

$$
\mathbf{x}_{k+1}=\left[\begin{array}{cc}
1 & \Delta T \\
0 & 1
\end{array}\right] \mathbf{x}_{k}+\left[\begin{array}{l}
0 \\
\omega
\end{array}\right]=A \mathbf{x}_{k}+B \omega,
$$

where $\omega$ is zero-mean white Gaussian noise with covariance $Q, B=\left[\begin{array}{ll}0 & 1\end{array}\right]^{T}$, and $\Delta T=t_{k+1}-t_{k}$. Note that our algorithm readily incorporates more complicated target dynamic models.

Based on the characterization of human UWB scatter as a point process, we model the multi-target multi-path scattering process as a mixture model, where each mixture component is a Gamma distribution associated with an individual human target. If there are $G$ human targets in the observation environment, where $G$ is known, the likelihood of the $N$ TOA measurements at time $k, Y^{k}=\left\{n_{j, k}\right\}_{j=1}^{N}$, is given by:

$$
p\left(Y^{k} \mid \Theta_{k}\right)=\prod_{j=1}^{N} \sum_{g=1}^{G} \pi_{k}^{g} f_{\Gamma}^{g}\left(\delta_{i, k}, \kappa_{k}^{g}, \theta_{k}^{g}\right),
$$

where $\Theta_{k}=\left\{\pi_{k}^{g}, \kappa_{k}^{g}, \theta_{k}^{g}\right\}_{g=1}^{G}$. Building upon the models, the detailed procedure of the Expectation Maximization Kalman Filter (EMKF) algorithm with its experimental validation can be found in [13].

\section{Tracking a Variable Number of Human TARGETS}

In a realistic environment, the number of targets will vary with time, as targets may go in and/or out of the observation volume. Additionally, the tracking system should also be able 
to handle clutter (e.g. non-human objects and false measurements) and missed detections (temporary occlusions), allowing for an appropriate segmentation process, simultaneously. Thus, it is necessary to develop a multi-target tracking (MTT) solution for these practical realities. The MTT technique should solve two types of data association problems: (1) all multipath scatter components must first be segregated according to their generating source (the observation-measurement association problem or the multipath scatter-cluster association problem); and then each scattering cluster must be associated to clusters from previous scans, thus tracking the UWB scattering response of putative human targets (the measurement-target or track assignment association problem).

We propose to use a Multiple Hypothesis Tracking (MHT) approach. However, unlike traditional MHT, that is, military radar and computer vision tracking applications, this problem has the additional complexity that targets are only observed indirectly via clusters of scattering path measurements. To incorporate these constraints, we adapt a recently developed Multiple-Hypothesis Tracking of Clusters (MHTC) method [11] that was originally developed for dynamic sorting and tracking of neural signals. This algorithm propagates various possibilities for how to assign measurements to existing targets and uses a delayed decision-making logic to resolve data association ambiguities. It also maintains several options, termed model hypotheses, for how to cluster the observations of each interval. This combination of clustering and tracking in a single solution enables MHTC to robustly maintain the identities of cluster-producing targets.

\section{A. MHTC Framework}

1) Hypothesis Terminology: We define two types of hypotheses in MHTC. A model hypothesis represents a possible clustering of the multipath observations, and is denoted by $\mathcal{M}_{m}$. Different models account for differing numbers of humans. The $l^{\text {th }}$ data association or track association hypotheses, $h_{l}=\left\{\tau_{l}, \nu_{l}, \phi_{l}\right\}, l=1, \ldots, L$, assigns each cluster in the given model hypothesis to a target or track (or marks it as spurious): The set $\tau_{l}$ contains the assignments of the model's clusters to known targets; $\nu_{l}$ contains the indices of the model's clusters that are identified as new human targets; and $\phi_{l}$ holds the indices of false clusters (spurious groupings of outliers, clutter, or similar clustering errors) in the current model. Note that $N_{\tau}, N_{\nu}$, and $N_{\phi}$ are the respective cardinalities of these sets and $G_{m}=N_{\tau}+N_{\nu}+N_{\phi}$.

We call the combination of a data association hypothesis and its parent model hypothesis a particular joint hypothesis at time $k, H_{l}^{k}=\left\{\mathcal{M}_{m(l)}, h_{l}\right\}$. The joint hypothesis $H_{l}^{k}$ thus postulates a complete set of data associations for time $k$, including the observation-measurement associations in $\mathcal{M}_{m(l)}$ and the measurement-target associations in $h_{l}$. A particular joint hypothesis is combined with its parent hypothesis $H_{\rho(l)}^{1: k-1}$ at time $k-1$ (we consider $\bar{M}$ model classes for each parent hypothesis) to define a global hypothesis, $H_{l}^{1: k}=\left\{H_{l}^{k}, H_{\rho(l)}^{1: k-1}\right\}$, which includes the full history of all model and data association hypotheses from time 1 through

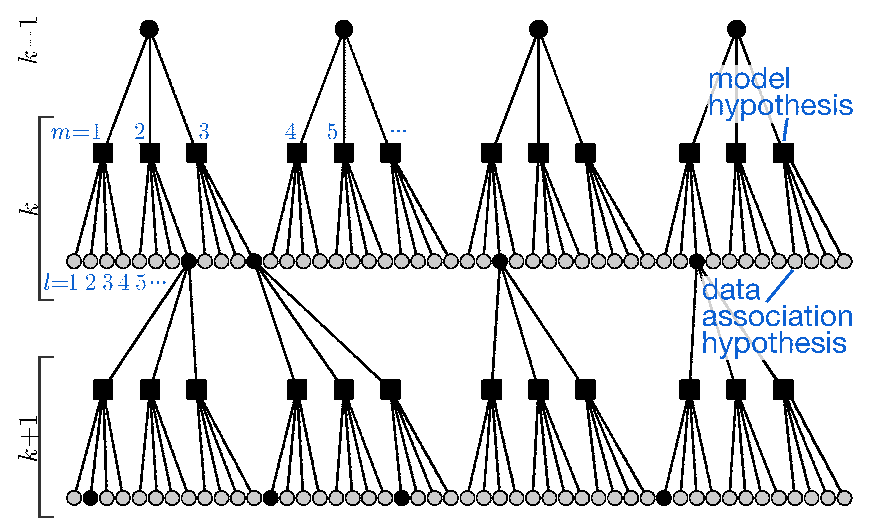

Fig. 2. MHTC hypothesis tree structure, illustrating the integration of model hypotheses into the traditional MHT framework, using $L=4$ and $\bar{M}=3$. Squares represent model hypotheses (i.e., clustering output) and black circles represent surviving data association hypotheses at each time step.

$k^{3}$ Finally, it is convenient to define $\Omega^{k}$ as the set of all $L$ surviving global hypotheses $\left\{H_{l}^{1: k}\right\}_{l=1}^{L}$ and all data $Y^{1: k}$, which thus provides all relevant measured and hypothesized information at time $k: \Omega^{k}=\left\{\left\{H_{l}^{1: k}\right\}_{l=1}^{L}, Y^{1: k}\right\}$.

2) Probability Models: Given a set of targets tracked in the parent hypothesis $H_{\rho(l)}^{1: k-1}$, the probabilities of the existence of tracked targets and location of new measurements in radar scan at time $k$ must be modeled. For simplicity, let us assume that the probability that the $j^{t h}$ existing target is detected (i.e., produces a multi-path cluster) is considered a Bernoulli trial with probability $\mathrm{P}_{\mathrm{d}, j}$. If the target is detected, the associated measurement is expected to appear near the target's predicted location with a Gaussian distribution, due to the nature of the Kalman filter applied. The numbers of new targets or false clusters appearing in a given time interval are each modeled by a Poisson distribution with respective rates $\lambda_{\nu}$ and $\lambda_{\phi}$. If a measurement originates from a new target or false cluster, it may arise anywhere in the observation volume $V$ with a uniform PDF. The parameters $\mathrm{P}_{\mathrm{d}, j}, \lambda_{\nu}$, and $\lambda_{\phi}$ are set by the user and may vary across scanning intervals.

\section{B. Hypothesis Tree Structure}

As shown in Figure 2, the MHTC algorithm extends the traditional MHT hypothesis tree to include model hypotheses as well as data association hypotheses. If $L$ global hypotheses exist/survive at time $(k-1)$ and we consider $\bar{M}$ model classes spawned for each of $L$ parent hypotheses, then $(L \bar{M})$ model hypotheses are formed at time $k$, each of which is optimized according to the EM procedure of Section III. By use of Murty's algorithm [15], only the $L$ best data association hypotheses are retained at time $k$ from $(L \bar{M})$ data association hypotheses, succeeded by the $(L \bar{M})$ model hypotheses.

\section{Overview of the MHTC Process}

This section summarizes the MHTC process of the combined clustering and multiple hypothesis tracking.

\footnotetext{
${ }^{3}$ The subscript $m(l)$ is used to indicate the index of the model or global hypothesis that is the parent of the $l^{t h}$ data association hypothesis; $\rho(m)$ indicates the parent global hypothesis of the $m^{t h}$ model hypothesis.
} 
Step 1. Initialize the EM and Gamma mixture model parameters for the EMKF algorithm in Section III.

Step 2. Given $\Omega^{k-1}$ and the measurement update from time $k-1$ (see Step 8), for each model hypothesis predict the target states (and their covariances) using the Kalman Filter dynamic update equations in the EMKF algorithm.

Step 3. For every parent hypothesis in $\Omega^{k-1}$, a set of candidate mixture model classes $\left\{\mathcal{M}_{m}\right\}$ are postulated, which will be used to cluster the current data $Y^{k}=\left\{n_{j, k}\right\}_{j=1}^{N}$, preprocessed by the MTI system and the CLEAN algorithm. A range of model classes are required primarily because the number of clusters $G_{m}$ is unknown, so that various model orders are attempted and each resulting model hypothesis is analyzed. To save computation in very unlikely model classes, one can calculate the probability of a model class and test it against a threshold $\beta$ :

$$
P\left(\mathcal{M}_{m} \mid H_{\rho(m)}^{1: k-1}, Y^{1: k-1}\right)>\beta .
$$

An expression for this probability, which depends on the probabilities of target detection, new targets, and false clusters, is provided in Table I. Model classes that do not pass this thresholding test are discarded.

Step 4. For each model class, optimize the assignment of scattering multipaths to individual model clusters, and optimize the cluster parameters using the EM algorithm of Section III. Note, to carry out this step, each model requires a set of "seed" clusters. The seed clusters are generated by starting with the clustering solution of the previous step, and then by adding or removing clusters according to the different hypotheses associated with each model. For example, new seeds are placed at the sensing boundaries to account for incoming targets; previous clusters are "split" into two clusters to allow for possible misclustering in the previous step; random seeds are added to account for spurious clusters, etc. (see [11] for more details).

Step 5. The evidence of each model hypothesis, $p\left(Y^{k} \mid \mathcal{M}_{m}, H_{\rho(m)}^{1: k-1}, Y^{1: k-1}\right)$ is calculated, possibly using Bayes Information Criterion (BIC) [16]. For computational savings, one could prune highly unlikely models.

Step 6. The core step in MHT generates the data association hypotheses, $h_{l}=\left\{\tau_{l}, \nu_{l}, \phi_{l}\right\}$. Murty's $L$-best ranked linear assignment algorithm is used to produce only the $L$ best data association hypotheses from each parent cluster hypothesis, obviating the need for full enumeration of all possible data associations.

Step 7. Suppose that a total of $\tilde{M}$ model hypotheses exist at this time, each of which has now spawned $L$ data association hypotheses. From the $(\tilde{M} L)$ hypotheses that have been generated, the most probable $L$ global hypotheses (consisting of a joint model and data association hypothesis) are selected. Evaluating each model and data association hypothesis together with its parent hypothesis $H_{\rho(l)}^{1: k-1}$, the probability of each new global hypothesis $P\left(H_{l}^{1: k} \mid Y^{1: k}\right)$ can be calculated, as detailed in Section IV-D. This step provides the set of best global hypotheses in $\Omega^{k}$.
Step 8. Finally, for each $H_{l}^{1: k} \in \Omega^{k}$, the hypothesized data associations $h_{l}$, along with the optimized parameters $\Theta_{m}^{k}$ of the corresponding model hypothesis, are used to update the Kalman Filter in Section III.

\section{MHTC Probabilities}

The key probability to be determined for MHTC is that of a global hypothesis given all collected data, $P\left(H_{l}^{1: k} \mid Y^{1: k}\right)$, which is the basis of the final hypothesis selection for time $k$ (in Step 8). The expression for this probability includes all relevant measures about the parent hypothesis, model hypothesis, and data association hypothesis. This global hypothesis probability may be expressed as

$$
P\left(H_{l}^{1: k} \mid Y^{1: k}\right) \approx \frac{1}{\mathcal{C}} \frac{\mathcal{P}_{1, l} \mathcal{P}_{2, l}}{\sum_{n \in \Gamma} \mathcal{P}_{1, n} \mathcal{P}_{2, n}} \mathcal{P}_{3} \mathcal{P}_{4} \mathcal{P}_{5},
$$

where $\mathcal{C}$ is a normalization constant, $\Gamma$ is the set of indices of all legal data association hypotheses given the model hypothesis $\mathcal{M}_{m(l)}$. The factors $\left(\mathcal{P}_{1, l}, \mathcal{P}_{2, l}\right.$, etc.) have natural interpretations for why they influence the global hypothesis probability and are described in Table I. The proof for Eq. (6), which entails a combination of Bayes' Rule, the chain rule, and BIC for approximating integrals, may be found in [11], along with a derivation for the expressions in Table I.

To calculate $L$-best data association hypotheses $\left\{h_{l}\right\}$ from each model hypothesis $\mathcal{M}_{m}$ in Step 6 of the MHTC algorithm, only the product $\left(\mathcal{P}_{1, l} \mathcal{P}_{2, l}\right)$ needs to be examined, as all other factors in (6) are identical for a given model hypothesis. Thus, we refer to this product as the data association hypothesis plausibility. To formulate the data association problem such that Murty's algorithm may be applied, we construct a cost matrix for the corresponding linear assignment problem of mapping current measurements to known targets (including the notions of new targets and false clusters), where the total cost of an assignment hypothesis is equivalent to using $\left(\mathcal{P}_{1, l} \mathcal{P}_{2, l}\right)$. See [11] for details.

\section{EXPERIMENTAL RESULTS}

To test the MHTC algorithm, UWB mono-static radar measurements were conducted by using a Time Domain PulsOn 210 monostatic UWB radar for time-varying number of human targets walking in and out of the radar observation volume. Figure 3(a) shows 547 unprocessed scans (each column represents the magnitude of a single scanned waveform over the sensing range, where the waveform magnitude is scaled from 0 in black to 10000 in white. Magnitudes over 10000 are clipped to 10000). Moving human targets' trajectories can be seen in Figure 3(a), while the horizontal patterns represent direct antenna coupling effects in the near range. Figure 3(b) show MTI responses of walking human scatters and the output of the CLEAN algorithm for the $360^{\text {th }}$ scan. The radar scanning period $\Delta T$ was $0.0786 \mathrm{sec} / \mathrm{scan}=(12.7 \mathrm{scans} / \mathrm{sec})^{-1}$, and the waveform sampling resolution was 41.33 ps with the range resolution of $0.0062 \mathrm{~m}$. The radar returns were processed using the CLEAN algorithm with $T_{\text {clean }}=5 \times 10^{4}$ to extract the amplitudes and TOAs of the scattering components. These 
TABLE I

FACTORS in GLOBAL Hypothesis PRobability

\begin{tabular}{rlll} 
& Definition & Expression for Model in Equation (6) & Interpretation \\
\hline $\mathcal{P}_{1, l}$ & $p\left(\left\{\hat{r}_{g}^{k}\right\}_{g=1}^{G_{m(l)}} \mid H_{l}^{1: k}, Y^{1: k-1}, \hat{\Theta}_{m(l)}^{k}\right)$ & {$\left[\prod_{(g, j) \in \tau_{l}} f_{\mathcal{N}}\left(\hat{r}_{g}^{k} \mid \hat{r}_{j}^{k \mid k-1}, \operatorname{cov}(\hat{r})_{j}^{k}\right)\right]\left[\prod_{g \in \nu_{l}} \frac{1}{V}\right]\left[\prod_{g \in \phi_{l}} \frac{1}{V}\right]$} & locations' likelihood under $h_{l}$ \\
$\mathcal{P}_{2, l}$ & $p\left(H_{l}^{k} \mid H_{\rho(l)}^{1: k-1}, Y^{1: k-1}\right)$ & $A_{m}\left[\prod_{j=1}^{J} f_{\mathcal{B}}\left(\delta_{j, l} \mid \mathrm{P}_{\mathrm{d}, j}\right)\right]\left(\lambda_{\nu}\right)^{N_{\nu}}\left(\lambda_{\phi}\right)^{N_{\phi}}$ & joint hypothesis prior ${ }^{a}$ \\
$\mathcal{P}_{3}$ & $P\left(\mathcal{M}_{m(l)} \mid H_{\rho(l)}^{1: k-1}, Y^{1: k-1}\right)$ & $\sum_{N_{\tau}=0}^{J}\left[\frac{\left(\lambda_{0}\right)^{G_{m}-N_{\tau}} e^{-\lambda_{0}}}{\left(G_{m}-N_{\tau}\right) !} \sum_{\delta \in \Upsilon} \prod_{j=1}^{J} f_{\mathcal{B}}\left(\delta_{j, l} \mid \mathrm{P}_{\mathrm{d}, j}\right)\right]$ & model hypothesis prior ${ }^{b}$ \\
$\mathcal{P}_{4}$ & $p\left(Y^{k} \mid \mathcal{M}_{m(l)}, H_{\rho: k-1}^{1:(l)}, Y^{1: k-1}\right)$ & see [16] for Bayes Information Criterion (or other approximation) & model evidence \\
$\mathcal{P}_{5}$ & $P\left(H_{\rho(l)}^{1: k-1} \mid Y^{1: k-1}\right)$ & same as Eq. (6), from previous time step & parent hypothesis probability \\
\hline
\end{tabular}

${ }^{a} A_{m}$ is a constant depending on the model class and does not require calculation. $f_{\mathcal{B}}$ is the Bernoulli distribution, and $\delta_{j, l}$ is an indicator variable of whether the $j^{\text {th }}$ target is tracked under the $l^{\text {th }}$ hypothesis $\left(j \in \tau_{l}\right)$.

${ }^{b} \lambda_{0}=\lambda_{\nu}+\lambda_{\phi} . J$ is the number of existing targets, $\boldsymbol{\delta}$ is a vector of indicator variables $\delta_{j}, j=1, \ldots, J$, and $\Upsilon$ is the set of all possible $\boldsymbol{\delta}$ for a given $J$.

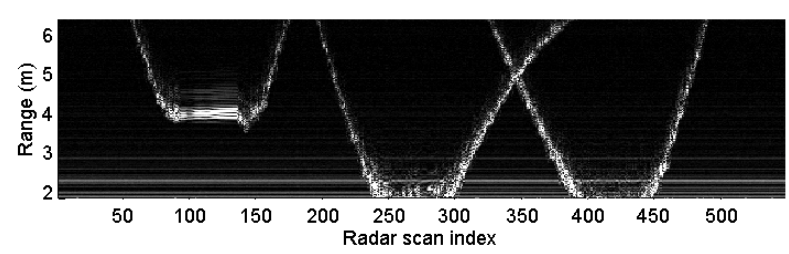

(a) Representation of 547 unprocessed scans.
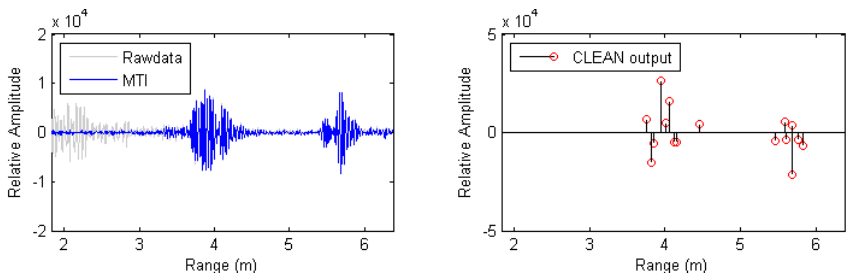

(b) Preprocessing for scan 360 .

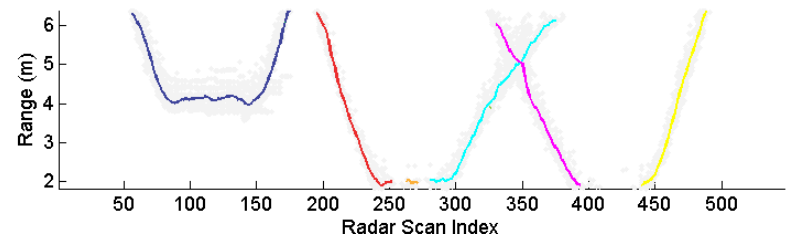

(c) Estimated human target ranges, overlaid on multipath TOAs.

Fig. 3. The MHTC results with changing numbers of human targets.

measurements were then processed using the MHTC algorithm with parameters of $\kappa=7.60, K=0.533 \mathrm{~m}, L=6$, $\mathrm{P}_{\mathrm{d}, j}=0.99, \lambda_{\nu}=0.01$, and $\lambda_{\phi}=0.0105$. Figure 3(c) shows that human targets are tracked for a variable number of human, even when two humans are walking cross over at around the $360^{\text {th }}$ scan.

\section{DiscuSSION AND CONCLUSION}

In [13] we introduced the novel observation that monostatic UWB radar multipath scatters from walking humans can be modeled as a point process. This allowed us to develop a rigorous method to track a fixed number of human targets. In this paper we extended this method to handle a variable number of targets, along with clutter and temporary occlusions. This extension is necessary to make our tracking approach applicable to realistic problems. The key to our extension was the novel formulation of a Multiple Hypothesis Tracking for Clusters (MHTC) procedure which allowed us to rigorously organize and select the complex data associations inherent in UWB multi-path scattering from multiple targets. Ongoing work seeks to improve our method to identify clutter in the UWB return signal, and to extend the approach to multiantenna configurations.

\section{REFERENCES}

[1] S. Chang, R. Sharan, M. Wolf, N. Mitsumoto, and J.W. Burdick, "UWB radar-based tracking of humans," in Proc. IEEE RadarCon 2009, Pasadena, CA, May 4-8, 2009.

[2] M. I. Skolnik, Radar Handbook, New York: McGraw Hill, 1970.

[3] P. Z. Peebles, Jr., Radar Principles, New York: Wiley, 1998.

[4] R. M. Narayanan, "Through-Wall Radar Imaging using UWB Noise Waveforms," J. Franklin Institute vol. 345, no. 6, pp. 659-678, Sept. 2008.

[5] C. Chang, and A. Sahai, "Object tracking in a 2D UWB sensor network," in Proc. $38^{\text {th }}$ Asilomar Conf. on Signals, Systems and Computers, vol. 1, pp. 1252-1256, 2004.

[6] R. A. Scholtz, D. M. Pozar, and W. Namgoong, "Ultra-Wideband Radio, EURASIP Journal of Applied Signal Processing, No. 3, 2005, pp. 252272. 5. G. Franceschetti, C.

[7] Y. Bar-Shalom and T. E. Fortmann, Tracking and Data Association, Academic Press Inc., Orlando, FL, 1988.

[8] S. S. Blackman and R. Popoli, Design and Analysis of Modern Tracking Systems, Artech House, Norwood, MA, 1999.

[9] G. W. Pulford, "Taxonomy of multiple target tracking methods," IEE Proceedings - Radar, Sonar \& Navigation, 152(5):291 304, 2005.

[10] E. F. Knott, J. F. Shaeffer, and M. T. Tuley, Radar Cross Section, $2^{\text {nd }}$ ed., Artech House, Norwood, MA, 1993.

[11] M. Wolf, "Target Tracking Using Clustered Measurements, with Applications to Autonomous Brain-Machine Interfaces," Ph.D. thesis, Department of Mechanical Engineering, California Institute of Technology, Pasadena, CA, June 2008.

[12] H. Hashemi, "Impulse response modeling of the indoor radio propagation channels," IEEE Trans. Select. Areas Commun., vol. 11, no. 7, Sep. 1993.

[13] S. Chang, N. Mitsumoto, J.W. Burdick, "An algorithm for UWB radarbased human detection," in Proc. IEEE RadarCon 2009, Pasadena, CA, May 4-8, 2009.

[14] S. M. Yano, "Investigating the ultra-wideband indoor wireless channel," in Proc. IEEE VTC Spring Conf., vol. 3, pp. 1200.1204, 2002.

[15] K. G. Murty, "An algorithm for ranking all the assignments in order of increasing cost," Operations Research, 16:682-687, 1968.

[16] J.L. Beck and K.-V. Yuen, "Model Selection Using Response Measurements: Bayesian Probabilistic Approach," J Engineering Mechanics, vol. 130, no. 2, pp. 192-203, 2004. 